\section{Cahiers de Narratologie}

Analyse et théorie narratives

$35 \mid 2019$

Le style comme événement

\title{
Jan Mukaroský, Écrits 1928-1946
}

\section{Marc Marti}

\section{(2) OpenEdition}

\section{Journals}

Electronic version

URL: https://journals.openedition.org/narratologie/9242

DOI: $10.4000 /$ narratologie.9242

ISSN: 1765-307X

\section{Publisher}

LIRCES

\section{Electronic reference}

Marc Marti, "JAN MUKAROSKÝ, É ÉRITs 1928-1946", Cahiers de Narratologie [Online], 35 | 2019, Online since 03 September 2019, connection on 21 September 2021. URL: http://journals.openedition.org/ narratologie/9242 ; DOI: https://doi.org/10.4000/narratologie.9242

This text was automatically generated on 21 September 2021.

Article L.111-1 du Code de la propriété intellectuelle. 


\title{
Jan Mukaroský, Écrits 1928-1946
}

\author{
Marc Marti
}

\section{REFERENCES}

Jan Mukaroský, Écrits 1928-1946, éditions des archives contemporaines, Paris, 2018, édition scientifique de John Pier

1 En France, si on fait exception de la reconnaissance de l'antériorité du formalisme russe dans les écrits de la seconde partie du XXe siècle, le structuralisme comme théorie littéraire a fini par s'imposer comme une sorte de production intellectuelle nationale, née schématiquement de la rencontre et de la combinaison théorique entre Roman Jakobson, Claude Levi-Strauss et Ferdinand de Saussure. Et comme l'écrit John Pier dans l'introduction, «le structuralisme français, en s'appuyant sur Saussure et les formalistes russes, a sauté la case du structuralisme tchèque ", souvent désigné par le groupe qui le représente, le Cercle Linguistique de Prague (CLP). L'ouvrage recensé dans les lignes qui suivent propose très justement d'offrir au lecteur francophone les écrits principaux de la figure majeure du CLP, Jan Mukaroský, grâce aux traductions de Jan Boutan, Xavier Galmiche, Kristýna Matisová et Laurent Vallance.

2 L'accès à ces textes représente bien plus que la résolution d'une lacune dans l'histoire des idées. En effet, contrairement à ce qui a été pensé pendant de nombreuses années, principalement par ignorance des écrits du groupe, le CLP n'est absolument pas un simple avatar du formalisme et encore moins un simple proto-structuralisme. L'introduction de John Pier est fondamentale pour comprendre la place et l'évolution historique de Mukarosvký qui « inaugure une conception dynamique et dialectique de la structure qui met en cause la nature des relations statiques entre la partie et le tout ».

3 En effet, les trois étapes de la réflexion du théoricien montrent une évolution vers la prise en compte du rapport entre l'œuvre, sa structure et son contexte historique et social. Dans un premier moment, le théoricien tchèque s'est attardé sur « l'organisation 
interne de l'œuvre littéraire en tant qu'artefact ou "œuvre-chose" » dont la cohérence repose sur la dominante.

4 Cependant, Mukarovský franchira une étape majeure en théorisant le dépassement de ce que l'on nommera plus tard la structure. Pour lui, l'œuvre d'art « n'est pas non plus un objet dont l'organisation représente une fin en soi », elle est prise, comme tout objet culturel, « dans un réseau de communication, mais à la différence des textes à fonction communicative, l'œuvre d'art est un signe autonome ». Cette idée du théoricien est sans doute la plus célèbre, bien que le concept ait été parfois mal saisi. En effet, dans «L'art comme fait sémiologique », l'idée est que « l'œuvre d'art est un fait social soutenu par un système de codes qui varie selon le contexte social et historique ». On retrouve ici une conception qui sera centrale dans la sémiologie, celle de la fonction esthétique qui ne relève pas uniquement du domaine de l'art, mais qui « se trouve dans toutes les sphères de la vie sociale (les produits de consommation, la mode, etc.)».

5 L'anhistoricisme du structuralisme est ainsi dépassé et l'on tend vers une théorie qui nous semble familière chez Roland Barthes sémiologue (étude de la publicité ou des Mythologies) ou chez Umberto Eco sémioticien et sa théorie interprétative.

6 La dernière phase théorique de Mukarosvký fut donc logiquement celle d'un questionnement sémiotique qui interroge la notion d'universaux. Il est résumé dans le titre de l'article «La valeur esthétique dans l'art peut-elle être universelle», qui s'interroge "sur la part du relatif et celle de l'universel dans la valeur esthétique ", avec un second questionnement esthétique incontournable, celui du rôle de l'individu.

7 Le premier article de Petr A. Bílek et Tomás Kubícek est intitulé «Définir le structuralisme tchèque ". Il rappelle les principaux apports du CLP à la théorie, en particulier l'ajout de la fonction esthétique du langage que reprendra Roman Jakobson à la fin des années cinquante, avec les six fonctions. Les auteurs rappellent aussi le contexte historique de la Tchécoslovaquie, où la censure idéologique de l'après-guerre a mis fin aux recherches structuralistes, au profit de la théorie " du reflet ", chère à la doxa politique du moment. L'addendum de John Pier en fin de texte précise cependant l'essaimage des continuateurs du CLP à travers le monde dès la fin des années quarante.

«Du structuralisme» présente le texte prononcé par Jan Mukarovský en 1946 lors d'une conférence à l'Institut des Études Slaves de Paris. Il s'agit d'une réflexion globale sur la façon d'entendre le concept de structure, dont la portée est absolument fondamentale et conditionne d'ailleurs une bonne partie des articles présentés à la suite dans l'ouvrage. On ne peut ici que se féliciter de la publication de ce texte en français qui démontre combien, dans ses propositions, le structuralisme tchèque se démarquait de l'anhistoricisme d'autres théories.

9 D'emblée, il est affirmé qu'on «ne peut tenir pour structure qu'un ensemble de composantes dont l'équilibre interne est constamment rompu et de nouveau recréé, et dont l'unité nous apparaît en conséquence comme un ensemble d'antagonismes dialectiques. Seule perdure l'identité de la structure au cours d'une période donnée, tandis que sa composition interne, la corrélation entre ses composantes, change constamment [...] Les significations qui passent [...] au premier plan ont une signification décisive pour le sens général de la structure artistique, qui change sans cesse, du fait de leur recomposition ».

De fait, la structure, telle que la définit Mukarovský, s'inscrit dans la perspective d'une histoire de l'Art : «[...] si une œuvre d'art doit être comprise comme une structure, il 
faut qu'elle soit perçue - et d'abord créée - sur le fond de certaines conventions artistiques (formules) fournies par la tradition artistique présente dans l'inconscient de l'artiste comme dans celui qui la perçoit ». S'y ajoute deux éléments particulièrement suggestifs. D'une part la notion d'influence est pensée comme un processus dialectique productif à l'intérieur des arts nationaux, sans qu'il n'y ait jamais de rapport de domination. D'autre part, la structure peut aussi être envisagée dans la relation que les arts entretiennent entre eux, c'est-à-dire " un équilibre fugace de relations ».

11 La fin de l'article précise le rôle de la fonction esthétique. Pour le théoricien tchèque, «il est évident que la fonction esthétique est loin de se cantonner au seul domaine de l'art, mais qu'elle est mêlée à tout le travail de l'homme et à toutes les manifestations de son existence. Elle est l'un des facteurs les plus importants qui forgent le rapport de l'être humain à la réalité [...]. C'est précisément parce qu'elle n'a pas de "contenu" univoque que la fonction esthétique devient "transparente", ne se montre pas hostile aux autres fonctions, mais les aide « [...] se manifestant dans l'art comme une fonction spécifique, la fonction esthétique aide l'être humain à dépasser ce qu'il y a d'unilatéral dans la spécialisation, qui appauvrit non seulement son rapport à la réalité, mais aussi ses possibilités d'action sur elle».

Dans «Le concept de tout dans la théorie de l'art ", conférence prononcée en 1945 au CLP, Mukarovský s'intéresse en fin de réflexion aux relations entre sciences sociales et structure. Selon lui, depuis le point de vue des sciences sociales, la structure pourrait « être qualifiée d'ensemble de normes; mais les normes ne doivent pas être comprises comme des règles statiques, mais comme des forces vivantes ».

13 "Contradictions dialectiques dans l'art moderne", de 1935 semble plus directement une application de la théorie de la structure à l'histoire littéraire, un texte dans lequel Mukarovský examine les rapports dialectiques des composantes à l'intérieur de la structure et leur jeu dynamique.

Le dernier article de la première partie est une réflexion majeure, qui fait de Mukarovský un pionnier de la sémiotique littéraire. Dans "L'art comme fait sémiologique ", un texte publié à Pragues en 1936, il énonce les cinq points de sa théorie sur le sujet, avec des propositions qui étaient révolutionnaires à l'époque, en particulier l'affirmation que l'œuvre d'art a un caractère de signe, que ce signe est autonome et que deux fonctions, communicative et autonome, constituent la dualité du signe.

15 La deuxième partie, «La poétique du texte », contient des études sur la littérature, qui reprennent d'une autre façon la théorie structuraliste. On retiendra en particulier, « La tradition de la forme ", article antérieur à celui sur le structuralisme et qui se pose la question de la nature de la forme à travers l'histoire littéraire, conçue soit comme technique artistique, soit comme expression de l'individualité ou soit comme phénomène possédant des principes internes tout en étant ouvert aux influences externes. L'intérêt de l'apport de Mukarovský est d'avoir intégré à la forme une perspective diachronique, en y incluant son évolution dans le temps, «son caractère ininterrompu ", son devenir dans le temps - idée que l'on retrouve dans l'article final de la troisième partie "L'individu dans l'art ».

16 La troisième partie, "L'esthétique de l'œuvre d'art » développe la fonction esthétique propre au structuralisme tchèque et que Mukarosvký avait défini dans son texte sur le structuralisme. L'introduction de Tomás Kubicek vient rappeler l'évolution du théoricien sur le sujet et son passage d'une théorie de l'objet (langue ou texte) vers une 
théorie du sujet interprétant. "L'esthétique de la langue », un texte de 1940, est un long essai de réflexion sur le concept et ses relations avec la langue. L'article suivant "Individu et évolution littéraire » (1943) est intéressant, dans le sens où, loin de rejeter «la question de l'auteur» comme on l'impute traditionnellement au structuralisme classique, il l'inclut au contraire à la théorie interprétative. Il se démarque de la vision positiviste psychologisante en considérant la personnalité comme un fait historique et dynamique. Cette ligne critique se poursuit dans l'article «Intentionnalité et nonintentionnalité dans l'art». Le postulat de départ annonce qu'il "y a de nombreuses choses dans l'œuvre d'art prise comme un tout et dans l'art en général qui échappent à l'intentionnalité et, dans certains cas, dépassent l'intention de l'artiste ». Après avoir parcouru les théories psychologiques, Mukarovský situe le phénomène du côté de la réception et conclut que «Intentionnalité et non-intentionnalité sont des phénomènes sémantiques et nullement psychologiques : l'unification sémantique de l'œuvre et la négation de cette unification ». Le phénomène renvoie à la fameuse tension dialectique définie aussi dans l'article sur le structuralisme, «la force qui unifie les différentes composantes de l'œuvre en un sens général [intentionnalité] mais aussi la force contraire qui vise à remettre en cause l'unité du sens général [non-intentionnalité] ».

"L'œuvre poétique comme ensemble de valeur », publié en 1932, propose d'analyser « la relation entre la valeur esthétique, qui seule est nécessaire et dominante, et toutes les autres valeurs ", pour lesquelles Mukarovský propose l'appellation de valeurs extraesthétiques. Il en découle la possibilité d'un double jugement de valeur de l'œuvre poétique, qui se fonde sur cette distinction. La réflexion n'est pas orientée uniquement vers une sociologie du texte, de sa réception et/ou de sa production. Ce qui intéresse aussi le théoricien, c'est de faire la différence entre l'expression communicative et celle à visée esthétique - idée qui est aussi exploitée dans l'article suivant « La dénomination poétique et la fonction esthétique de la langue ». Mukarovský démontre que l'extraesthétique est au service de l'esthétique. Le problème rejoint celui des fonctions attribuées au texte, qui dépendent de l'usage qu'un groupe lui assigne. Ainsi, «la fonction extra-esthétique de la poésie n'est pas à proprement parler un problème de poétique, mais de sociologie de la poésie » à entendre ici comme sa portée et son usage social.

Les deux articles «La norme esthétique » et "La valeur esthétique dans l'art peut-elle être universelle " pose la même question, celle en quelque sorte de la nature de l'art. Pour Mukarovský, la norme relève de la fonction sociale, dont elle organise la réalisation. Il la distingue de la règle, qui en est simplement la codification linguistique. La norme est plus large, considérée ici comme un point d'orientation destinée à être dépassé par les tendances nouvelles qui s'expriment dans l'art. La norme peut être analysée en plusieurs catégories: celle qu'introduisent les matériaux utilisés; les "règles techniques ", comme les formes traditionnelles ou la loi des genres; les normes pratiques relevant de l'ordre religieux, politique, social, toutes étrangères à la fonction esthétique comme vu précédemment. Quant à la valeur universelle, Mukarovský établit que finalement elle est sans doute issue du devenir historique, comme «une énergie constamment vivante ", tout en se posant la question de la possibilité d'étendre ce postulat (en l'adaptant) aux autres espèces de valeurs universelles. 